\title{
Can we reduce the workload of mammographic screening by automatic identification of normal exams with artificial intelligence? A feasibility study
}

\author{
Alejandro Rodriguez-Ruiz ${ }^{1,2}$ - Kristina Lång ${ }^{3}$ - Albert Gubern-Merida ${ }^{2}$ Jonas Teuwen ${ }^{1}$ - Mireille Broeders ${ }^{4,5}$. \\ Gisella Gennaro ${ }^{6}$. Paola Clauser ${ }^{7}$ - Thomas H. Helbich $^{7}$ - Margarita Chevalier ${ }^{8}$ - Thomas Mertelmeier $^{9}$. \\ Matthew G. Wallis ${ }^{10} \cdot$ Ingvar Andersson ${ }^{11}$. Sophia Zackrisson ${ }^{12} \cdot$ Ioannis Sechopoulos ${ }^{1,5} \cdot$ Ritse M. Mann ${ }^{1}$
}

Received: 13 January 2019 / Revised: 12 March 2019 / Accepted: 20 March 2019 /Published online: 16 April 2019

(C) The Author(s) 2019

\begin{abstract}
Purpose To study the feasibility of automatically identifying normal digital mammography (DM) exams with artificial intelligence (AI) to reduce the breast cancer screening reading workload.

Methods and materials A total of $2652 \mathrm{DM}$ exams ( 653 cancer) and interpretations by 101 radiologists were gathered from nine previously performed multi-reader multi-case receiver operating characteristic (MRMC ROC) studies. An AI system was used to obtain a score between 1 and 10 for each exam, representing the likelihood of cancer present. Using all AI scores between 1 and 9 as possible thresholds, the exams were divided into groups of low- and high likelihood of cancer present. It was assumed that, under the pre-selection scenario, only the high-likelihood group would be read by radiologists, while all low-likelihood exams would be reported as normal. The area under the reader-averaged ROC curve (AUC) was calculated for the original evaluations and for the pre-selection scenarios and compared using a non-inferiority hypothesis.

Results Setting the low/high-likelihood threshold at an AI score of 5 (high likelihood $>5$ ) results in a trade-off of approximately halving (-47\%) the workload to be read by radiologists while excluding 7\% of true-positive exams. Using an AI score of 2 as threshold yields a workload reduction of $17 \%$ while only excluding $1 \%$ of true-positive exams. Pre-selection did not change the average AUC of radiologists (inferior 95\% CI >- 0.05) for any threshold except at the extreme AI score of 9.

Conclusion It is possible to automatically pre-select exams using AI to significantly reduce the breast cancer screening reading workload.
\end{abstract}

Ritse M. Mann

Ritse.Mann@radboudumc.nl

1 Department of Radiology and Nuclear Medicine, Radboud University Medical Center, PO Box 9101, 6500 HB Nijmegen, The Netherlands

2 ScreenPoint Medical BV, Stationplein 26, 6512 AB Nijmegen, The Netherlands

3 Institute for Biomedical Engineering, ETH Zurich, Gloriastrasse 35, 8092 Zürich, Switzerland

4 Department for Health Evidence, Radboud University Medical Center, P.O. Box 9101, 6500 HB Nijmegen, The Netherlands

5 Dutch Expert Centre for Screening (LRCB), Wijchenseweg 101, 6538 SW Nijmegen, The Netherlands

6 Veneto Institute of Oncology (IOV)-IRCCS, via Gattamelata 64, 35128 Padua, Italy
7 Department of Biomedical Imaging and Image-Guided Therapy, Division of Molecular and Gender Imaging, Medical University of Vienna, Waehringer Guertel 18-20, 1090 Vienna, Austria

8 Medical Physics Group, Radiology Department, Faculty of Medicine, Universidad Complutense de Madrid, Pza. Ramón y Cajal s/n, 28040 Madrid, Spain

9 Siemens Healthcare GmbH, Diagnostic Imaging, X-Ray Products, Technology \& Concepts, Siemensstr. 3, 91301 Forchheim, Germany

10 Cambridge Breast Unit and NIHR Biomedical Research Unit, Box 97, Cambridge University Hospitals NHS Foundation Trust, Cambridge Biomedical Campus, Hills Road, Cambridge CB2 0QQ, UK

11 Unilabs Breast Center, Skåne University Hospital, Jan Waldenströms gata 22, SE-20502 Malmö, Sweden

12 Diagnostic Radiology, Department of Translational Medicine, Lund University, Skåne University Hospital, SE-20502 Malmö, Sweden 


\section{Key Points}

- There is potential to use artificial intelligence to automatically reduce the breast cancer screening reading workload by excluding exams with a low likelihood of cancer.

- The exclusion of exams with the lowest likelihood of cancer in screening might not change radiologists' breast cancer detection performance.

- When excluding exams with the lowest likelihood of cancer, the decrease in true-positive recalls would be balanced by a simultaneous reduction in false-positive recalls.

Keywords Mammography $\cdot$ Breast cancer $\cdot$ Screening $\cdot$ Deep learning $\cdot$ Artificial intelligence

\begin{tabular}{ll}
\multicolumn{2}{l}{ Abbreviations } \\
AI & Artificial intelligence \\
AUC & Area under the curve \\
BI-RADS & Breast imaging reporting and data system \\
CAD & Computer-aided detection \\
CI & Confidence interval \\
DM & Digital mammography \\
PoM & Probability of malignancy \\
ROC & Receiver operating characteristic
\end{tabular}

\section{Introduction}

Population-based screening programs with digital mammography (DM) reduce mortality from breast cancer due to the earlier detection of the disease $[1,2]$, but their efficiency is continuously under discussion [3, 4]. False-positive findings [5] lead to negative effects such as unnecessary workup, participant anxiety and reluctance to re-attend screening, as well as a reduction in cost-effectiveness [6]. On the other hand, since the program-based sensitivity of screening is approximately 75\% [7], false-negative findings may lead to false reassurance and ultimately a delayed cancer detection. One of the reasons why mammographically visible cancers are missed is the low prevalence of cancer (approximately, 10 per thousand) in a screening population $[8,9]$.

Computer-aided detection (CAD) systems to improve mammography reading have been used since the beginning of this century. However, so far no study has found any direct improvement in screening outcomes, likely because of the low specificity of these traditional CAD systems $[10,11]$. The recent breakthrough in artificial intelligence (AI) performance, based on the use of deep learning algorithms, is now closing the gap between human and computer performance in many applications related to medical imaging [12]. Novel AI systems may, therefore, be able to improve the performance and efficiency of population-based screening programs [13]. For mammography evaluation, deep learning-based systems have demonstrated a stand-alone performance as good as radiologists [14], as well as a significant improvement of radiologists' breast cancer detection accuracy when used for decision support [15]. However, this radiologist-like performance may enable other uses of AI for mammography evaluation in screening. Of particular interest are approaches aimed at reducing workload, considering the increasing scarcity of (breast) radiologists in some countries [16-18].

In this work, we explore the possibility of using an AI system to pre-select likely-normal mammograms. This was done using an AI system that provides an exam-based score denoting the likelihood of cancer present in the mammogram. We analyzed the effects on performance of excluding exams with a low score (i.e., low likelihood of cancer present) from human reading, which would reduce the screening workload for radiologists and increase the cancer prevalence in the actually evaluated images.

\section{Materials and methods}

\section{Data and population characteristics}

Digital mammograms were collected from nine previously performed multi-reader multi-case (MRMC) observer studies [19-26]. The review board at each institution waived local ethical approval and informed consent or approved the use of the anonymized patient data for retrospective research.

All the datasets of the MRMC studies were enriched with exams with cancer. The ground truth, in terms of cancer present, benign lesion present, or absence of abnormalities, of each DM exam, was confirmed by histopathology and/or at least 1 year of follow-up. During each MRMC study, each DM exam was evaluated by multiple breast radiologists who provided malignancy scores for each exam (BI-RADS and/or level of suspicion).

In total, 2654 exams (653 with cancer, 768 with benign lesions, 1233 normal) and readings by 101 radiologists $(52 \%$ from the USA and $48 \%$ from Europe) were gathered (yielding 28,296 independent exam interpretations). Approximately, half the exams were from screening and half from clinical practice. Detailed information about the tumor histology was not available. The DM images were acquired with devices from four different vendors (Siemens Healthineers; Hologic Inc.; General Electric Healthcare; Sectra Mamea) across seven 
different countries. Further details of these nine studies have previously been reported elsewhere [14].

\section{Artificial intelligence system}

For this study, we used an AI system dedicated to breast cancer detection in DM and digital breast tomosynthesis (Transpara 1.4.0, Screenpoint Medical BV). The system uses deep learning convolutional neural networks, feature classifiers, and image analysis algorithms to detect calcifications and soft tissue lesions in two different modules [14]. Based on these detected lesions and overall exam appearance, the AI system assigns an exam-based integer score denoting the likelihood that cancer is present in the exam (hereafter AI score, also known as Transpara Score). This AI score ranges between 1 and 10 (10 means high likelihood that a cancer is present in the exam). The AI score is calibrated so that approximately the same number of normal exams (10\% of the total) is assigned to each AI score category. In a population with low prevalence of cancer (where most exams are normal), such as a screening population, it may therefore be expected that approximately $10 \%$ of the total exams are in each category. In a screening population, the $10 \%$ of exams scored 1 are predicted to have the lowest risk of harboring cancer (because category 1 has the lowest incidence of exams with cancer), while the $10 \%$ of exams scored 10 have the highest risk of harboring cancer (because category 10 contains the largest fraction of exams with cancer). Since the calibration of the AI score is performed only with screening mammograms without abnormalities, the AI score is independent to the composition of the datasets. However, the fact that all datasets used for this study were enriched with cancers implies that the found distribution of AI scores in our study is skewed towards higher numbers, since it should be expected that cancer cases are not evenly distributed over AI categories.

The AI system was trained, validated, and tested using an external database representative of screening containing over 9000 mammograms with cancer (one-third of which are presented as lesions with calcifications) and 180,000 mammograms without abnormalities. The AI score was also calibrated with this external database, using only the normal mammograms. The mammograms used in this study have never been used to train, validate, or test the algorithms. The mammograms originate from devices from four different vendors (Hologic; Siemens; General Electric; Philips) and institutions across Europe, USA, and Asia.

\section{Automated pre-selection of cases}

For this study, the distribution of the normal exams and those containing benign or malignant lesions according to the ground truth was computed as a function of the AI score. To divide the exams into two groups (excluded and pre-selected for evaluation), we varied the threshold dividing these two groups across all possible AI scores, i.e., from 1 to 9. Consequently, the pre-selection scenarios included exams-tobe-evaluated as those with scores greater than 1, 2, 3, 4, 5, 6, 7, 8 , or greater than 9 (equivalent to only pre-selected category 10). For each threshold, the characteristics of the exams in both groups were analyzed.

Under the pre-selection scenarios, we assumed that readers would only evaluate exams in the pre-selected group (high likelihood of cancer present), whereas exams in the lowlikelihood group would automatically be assigned a "normal" classification. Workload reduction throughout the text is therefore expressed in terms of the number of exams that have to be read by the screening radiologists. Given the calibration of the AI system, a pre-selection threshold of 5, for instance, means that half of the exams in a screening program would be excluded from human reading. An estimation of how radiologists' performance would change after pre-selection was calculated by a posteriori modification of the original radiologists' scores: for the exams in the excluded group, all the radiologists' scores were automatically modified to the lowest possible value (e.g., 1). This implies that we assumed invariance in human behavior for the pre-selected mammograms that were above the threshold and therefore should be evaluated.

\section{Statistics}

The breast cancer detection accuracy of radiologists in the original scenario was compared with the simulated preselection scenario with a non-inferiority null hypothesis [27-31] based on the differences in the average area under the receiver operating characteristic curve (AUC). The noninferiority margin was set at 0.05 in this study. Non-inferiority was concluded when the AUC difference "pre-selection scenario" - "original reading" was greater than 0 and the lower limit of the $95 \%$ confidence interval (CI) of the difference was greater than the non-inferiority margin. Confidence intervals were Bonferroni-corrected for multiple comparisons.

To obtain the average AUC across all our data, we used the public-domain iMRMC software for analysis (version 4.0.0, Division of Imaging, Diagnostics, and Software Reliability, OSEL/CDRH/FDA, Silver Spring, MD) [29, 30], which can handle not fully crossed study designs, such as the split-plot design resulting when pooling the nine datasets from this study $[32,33]$. The reader-averaged ROC curves were created by averaging the reader-specific non-parametric (trapezoidal) curves along lines perpendicular to the chance line [34]. This average is area-preserving; its AUC is equal to the readeraveraged non-parametric AUCs. The analysis was not done per dataset, given the homogeneous performance of the AI system across datasets seen in Rodriguez-Ruiz et al [14]. We 
therefore assumed that no differences per dataset would be present in this study.

\section{Results}

\section{Performance of the Al system}

The distributions of DM exams as a function of AI score are shown in Fig. 1 (for each type of exam according to ground truth: a, normal; b, cancer; c, benign). As expected, normal exams are distributed evenly across AI scoring categories, with an average of $10.0 \%$ of normal exams per category (range 7.2-14.9\%). For the exams containing cancer, 72.5\% are categorized within the highest cancer-present likelihood category (10), whereas $95.1 \%$ lie in the categories 5-10. In comparison, only $27 \%$ of exams containing benign lesions are in category 10. An example of an exam with cancer that was assigned a low AI score is shown in Fig. 2.

\section{Automated pre-selection of cases}

The proportion and type of exams that would be excluded from reading after a pre-selection of exams using different pre-selection thresholds are depicted in Fig. 3. The trade-off between reducing screening reading workload (e.g., excluding normal exams) and excluding exams containing cancer from the reading is shown: halving the workload of screening ( $-47 \%$ of screening exams) can be achieved if only exams with scores higher than 5 are read, at the expense of excluding $7 \%$ of cancer exams. With a threshold of 2 for pre-selection, for instance, only $1 \%$ of exams containing cancer are excluded but the reading workload is reduced by up to $17 \%$. Simultaneously, these thresholds would reduce the cases containing benign lesions by $27 \%$ and $5 \%$, respectively, thus reducing the number of false-positive recalls substantially.

After the pre-selection of exams, assuming invariance of reader behavior, the average breast cancer detection performance (AUC) of the radiologists did not change. The AUC for the reading of the pre-selection population was noninferior to the AUC of the original population. This was significant (lowest Bonferroni-corrected 95\% CI >-0.05, AUC differences were less than $1 \%$, see Fig. 4) for all possible thresholds except for 9, when only exams in the highest cancer-present likelihood category (10) would be evaluated (low $95 \% \mathrm{CI}=-0.052$, AUC decreased by $2 \%$ ).

\section{Discussion}

In this work, we have evaluated the feasibility of using an AI system to automatically discriminate between screening mammography exams that have a higher and a lower likelihood

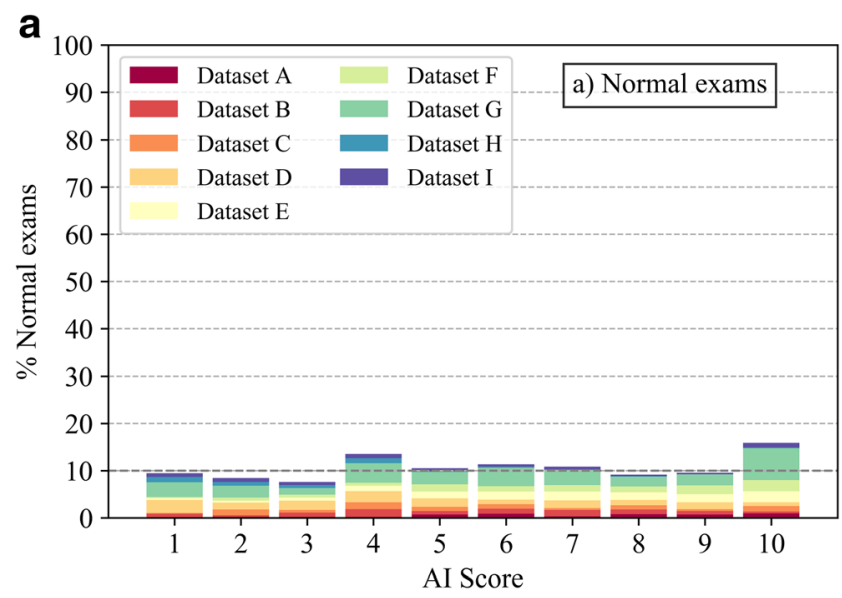

b

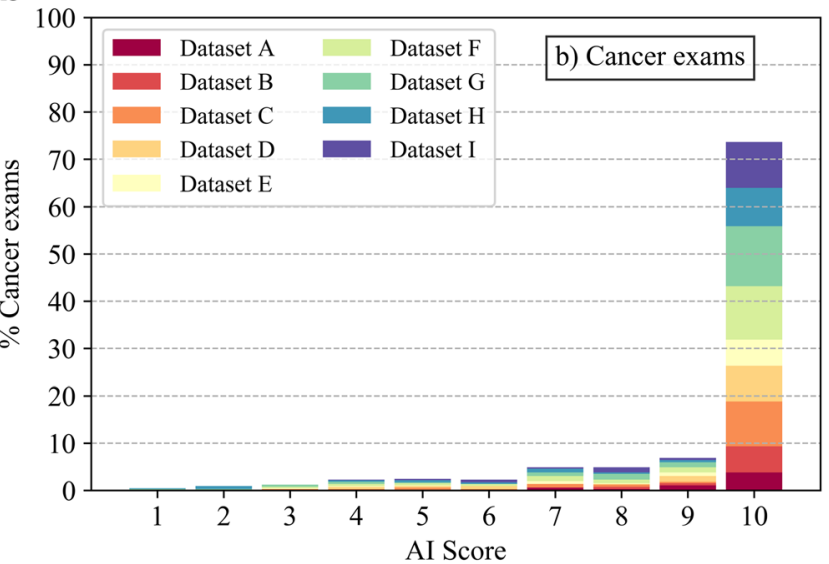

C

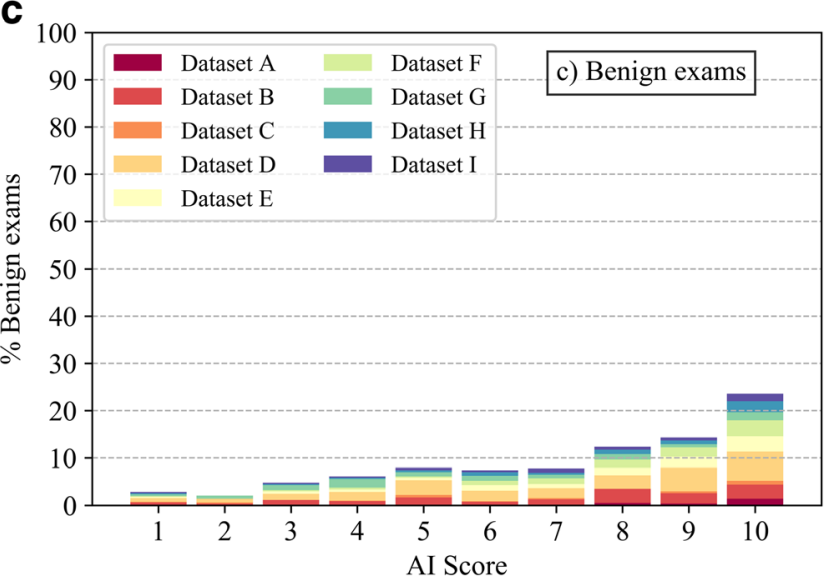

Fig. 1 Distribution of normal (a), cancer (b), and benign exams (c) as a function of AI score, representing the likelihood of cancer present (1-10, 10 means high likelihood of cancer present). The contribution of each dataset to the overall percentage of exams is shown

that cancer is present. Our study shows that, in some situations, it could be a possibility to exclude exams with a lower AI score from human evaluation since the cancer prevalence in these exams is much lower than in an unselected population, thus potentially reducing the reading workload for radiologists. 


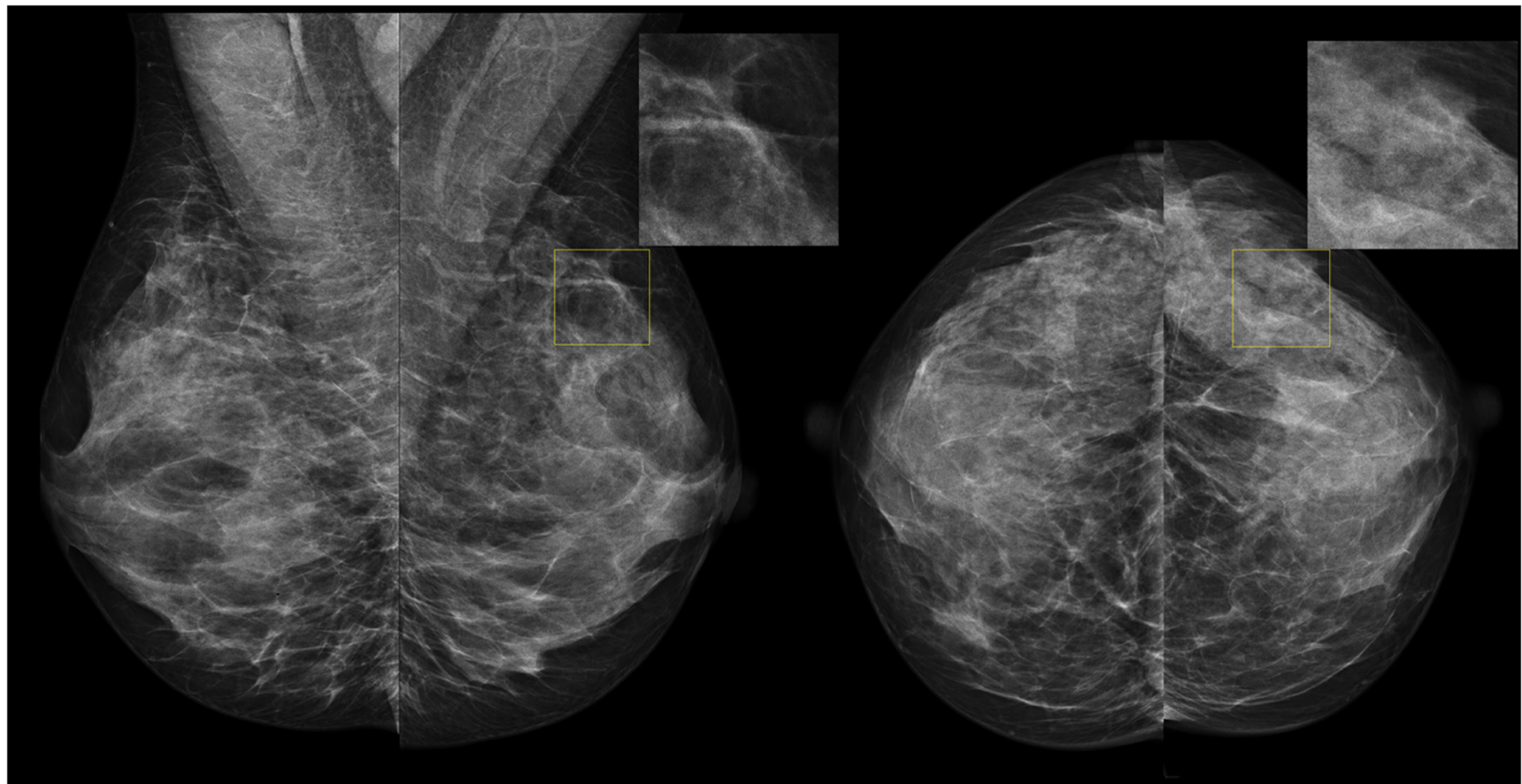

Fig. 2 An example of the nine exams in our study that contained cancer but were assigned an AI score of 1 or 2 , the lowest cancer-present likelihood categories. None of the 6 radiologists recalled this exam during the original MRMC study (read without priors), suggesting that the cancer visibility with mammography is poor in these exams (and in fact, the cancer may have been detected by other means)
If such a pre-selection scenario is to be considered, our results point to a trade-off between reducing workload and risking to exclude exams with cancer, which depends on the threshold chosen to create the two groups. On the other hand, the optimal threshold would likely be dependent on local practices and necessities. With a low threshold, we observed a relatively safe scenario with an approximate reduction of $17 \%$ in workload at the expense of excluding $1 \%$ of exams with cancer from reading (at a cancer detection rate in screening of approximately $6 / 1000$, the cancer prevalence in this group would be approximately 0.3 per 1.000 ). With a threshold set at an AI score of 5, the workload reduction increases to $47 \%$, at an expense of $7 \%$ of cancers. Nevertheless, the exams with cancer in this study do not only originate from screening but also from clinical practice, and it is reported that some cancers in the original reader study were detected by other means such as palpation, ultrasound or breast tomosynthesis [19-26]. This likely means that the reported percentage of excluded exams containing cancer in this study might be overestimating the actual exclusion proportion of screendetected cancers. A limitation of our study is that we cannot analyze the abovementioned results per detection mode (screening or clinical), per histopathological type of cancers or per breast density, because this information is not available from the original studies.

Our results suggest that pre-selection of exams does not lead to a reduction of the overall detection performance of radiologists, with the AUC varying by less than $1 \%$. This supports the theory that cancers missed by AI are also missed by radiologists, probability due to their low mammographic visibility.

We assumed invariance in reading of the pre-selected mammograms. However, in actual screening practice, there might be several factors affecting radiologists' scoring of preselected exams. The higher prevalence of cancer in the preselected cohort might lead to a higher sensitivity for breast cancer, as in practice, it is easier to detect abnormalities when their frequency is relatively high [8]. Likewise, it would be

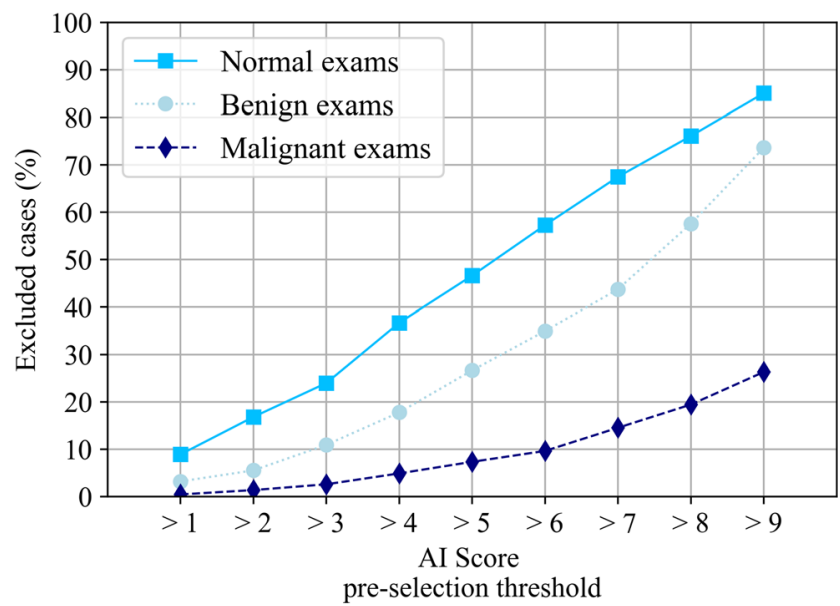

Fig. 3 Proportion (\%) of exams that would be excluded from the final sample to be evaluated by the radiologists, using all possible AI scores as thresholds values for pre-selection for reading 


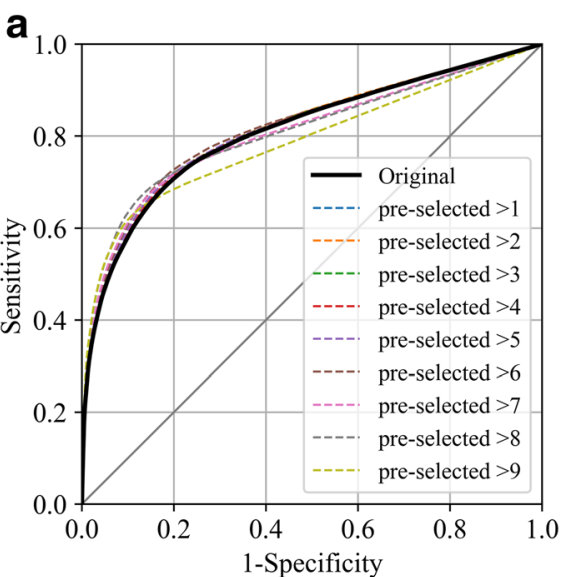

b

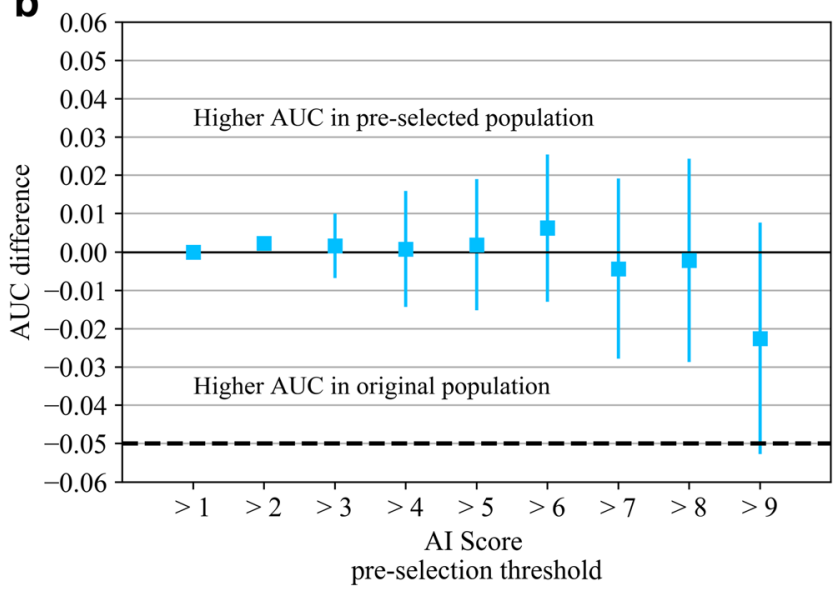

Fig. 4 ROC curves (a) and change (b) in AUC values of the average of radiologists in the original population, as well as in all possible preselected populations (using all possible AI scores as threshold values for pre-selection for reading; if the case is not pre-selected, the radiologist score is converted to the lowest possible cancer suspicion score for the MRMC study). 95\% confidence intervals are Bonferroni-corrected

interesting to investigate whether reading the pre-selected group of mammograms in a specific order, e.g., from higher to lower AI score, is also of added value (as cancer prevalence increases with increasing AI score). It would also be possible to increase the recall rate in the pre-selected cohort on purpose (by lowering the recall threshold), in order to counter-balance the exclusion of the cancer-containing mammograms with a low AI score. The fact that more benign lesions are excluded likely makes this possible without increasing the overall recall rate.

The exclusion of cases with benign lesions likely improves the specificity of screening, thus reducing possible harms associated with false-positive recalls. The similarity of ROC curves after exclusion of cases suggest indeed that the negative effect of dismissing exams with cancer is partially balanced by dismissing also false-positive assessments. However, it may be assumed that most benign abnormalities with a relatively low AI score will be lesions that are classified as certainly benign by breast radiologists without biopsy; therefore, the effect on the fraction of women that undergoes biopsy for benign lesions may be smaller.

While in this study we propose automatic labeling of mammograms that would never be read by human radiologists, an alternative possibility is to use the automatically created groups of exams to differentiate cases that need double reading, from cases for which single reading is sufficient. Such a strategy may be valuable for e.g. European screening programs, where double reading is a practice, and may be of special interest for programs that use breast tomosynthesis as the imaging technique for screening, because of the longer reading time per case $[25,35]$. Obviously, the effects of such stratification should be further evaluated.

Improvements of the computer system, such as inclusion of temporal information from prior exams, will presumably further enhance the pre-selection, as the current system only uses information from the current DM exams. Evaluation of other systems and versions should be regularly performed considering the rapid speed of evolution in the field of machine learning.

Our study had several limitations. The used datasets were not obtained from screening, but were enriched with cancer cases. The exams were not double-read, as is common practice in screening in Europe, but independently read by multiple radiologists per case. The mix of screening and clinical data may have also introduced cancers that have different characteristics from screen-detected cancers, which might bias our results. Since we have no histological characteristics of the tumors, we cannot be certain of the impact of the cancers that were excluded from human reading based upon the AI score on women's health. However, because mass screening per se is a balance between benefits, harms, and costs for the society, pre-selection of possibly abnormal and definitively normal cases may be a valid alternative for current screening practice. Further testing of such a pre-selection scenario in real screening populations is required to validate our findings in terms of the effect on recall rates, true-positive and false-positive screening assessments, and interval cancer rates.

In conclusion, we present a new strategy to reduce the reading workload in mammography-based breast cancer screening programs which do not appear to decrease the detection performance of radiologists, by using an AI system to automatically pre-select exams for radiologist evaluation while excluding those exams which have a low likelihood of harboring cancer from human reading.

Acknowledgements The authors would like to thank Dr. Brandon Gallas, Dr. Weijie Chen, and Mr. Qi Gong (Division of Imaging, Diagnostics, and Software Reliability, OSEL/CDRH/FDA, Silver Spring, MD, USA) for their help in implementing the statistical methods of the study with their iMRMC software (https://github.com/DIDSR/iMRMC). We would also like to thank all the radiologists involved in the reader studies whose results were used in this work and ScreenPoint Medical for providing their software for this research. 
Funding The authors state that this work has not received any funding.

\section{Compliance with ethical standards}

Guarantor The scientific guarantor of this publication is Ritse Mann.

Conflict of interest The authors of this manuscript declare relationships with the following companies:

The authors KL, PC, TH, TM, SZ, IS, and RM of this manuscript declare relationships with Siemens Healthineers (Erlangen, Germany): $\mathrm{TM}$ is an employee, $\mathrm{KL}, \mathrm{PC}, \mathrm{TH}, \mathrm{SZ}$, IS, and RM received research grants.

The authors AR, AG, and RM declare relationships with ScreenPoint Medical BV (Nijmegen, Netherlands): AR and AG are employees, RM is an advisor.

Statistics and biometry Dr. Brandon Gallas, Dr. Weijie Chen, and Mr. Qi Gong (Division of Imaging, Diagnostics, and Software Reliability, OSEL/CDRH/FDA, Silver Spring, MD, USA) kindly provided statistical advice for this manuscript.

One of the authors has significant statistical expertise.

No complex statistical methods were necessary for this paper.

Informed consent Written informed consent was waived by the Institutional Review Board.

Ethical approval Institutional Review Board approval was obtained.

Study subjects or cohorts overlap Some study subjects or cohorts have been previously reported in ("Stand-alone artificial intelligence for breast cancer detection in mammography: Comparison with 101 radiologists" by A. Rodriguez-Ruiz et al 2018, accepted in December 2018, Journal of the National Cancer Institute).

\section{Methodology}

- retrospective

- experimental

- multicenter study

Open Access This article is distributed under the terms of the Creative Commons Attribution 4.0 International License (http:// creativecommons.org/licenses/by/4.0/), which permits unrestricted use, distribution, and reproduction in any medium, provided you give appropriate credit to the original author(s) and the source, provide a link to the Creative Commons license, and indicate if changes were made.

\section{References}

1. Smith RA, Cokkinides V, Brooks D, Saslow D, Brawley OW (2010) Cancer screening in the United States, 2010: a review of current American Cancer Society guidelines and issues in cancer screening. CA Cancer J Clin 60:99-119

2. Broeders M, Moss S, Nyström L et al (2012) The impact of mammographic screening on breast cancer mortality in Europe: a review of observational studies. J Med Screen 19:14-25

3. Independent UK Panel on Breast Cancer Screening (2012) The benefits and harms of breast cancer screening: an independent review. Lancet 380:1778-1786

4. Welch HG, Prorok PC, O’Malley AJ, Kramer BS (2016) Breastcancer tumor size, overdiagnosis, and mammography screening effectiveness. N Engl J Med 375:1438-1447
5. Breast Cancer Surveillance Consortium (BCSC) Performance measures for $1,838,372$ screening mammography examinations from 2004 to 2008 by age-based on BCSC data through 2009 . National Cancer Institute. Available via http://www.bcsc-research. org/statistics/performance/screening/2009/perf age.html. Accessed 29 Sep 2017

6. Brewer NT, Salz T, Lillie SE (2007) Systematic review: the longterm effects of false-positive mammograms. Ann Intern Med 146: $502-510$

7. Karssemeijer N, Bluekens AM, Beijerinck D et al (2009) Breast cancer screening results 5 years after introduction of digital mammography in a population-based screening program. Radiology 253:353-358

8. Evans KK, Birdwell RL, Wolfe JM (2013) If you don't find it often, you often don't find it: why some cancers are missed in breast cancer screening. PLoS One 8:e64366

9. Huynh PT, Jarolimek AM, Daye S (1998) The false-negative mammogram. Radiographics 18:1137-1154 quiz 1243-1134

10. Fenton JJ, Taplin SH, Carney PA et al (2007) Influence of computer-aided detection on performance of screening mammography. N Engl J Med 356:1399-1409

11. Lehman CD, Wellman RD, Buist DS et al (2015) Diagnostic accuracy of digital screening mammography with and without computer-aided detection. JAMA Intern Med 175:1828-1837

12. Litjens G, Kooi T, Bejnordi BE et al (2017) A survey on deep learning in medical image analysis. Med Image Anal 42:60-88

13. Trister AD, Buist DSM, Lee CI (2017) Will machine learning tip the balance in breast cancer screening? JAMA Oncol. https://doi. org/10.1001/jamaoncol.2017.0473

14. Rodriguez-Ruiz A, Lång K, Gubern-Merida A et al (2019) Standalone artificial intelligence for breast cancer detection in mammography: comparison with 101 radiologists. J Natl Cancer Inst. https:// doi.org/10.1093/jnci/djy222

15. Rodríguez-Ruiz A, Krupinski E, Mordang J-J et al (2018) Detection of breast cancer with mammography: effect of an artificial intelligence support system. Radiology 181371

16. Rimmer A (2017) Radiologist shortage leaves patient care at risk, warns royal college. BMJ 359

17. National Health Institutes England, Public Health England, British Society of Breast Radiology, Royal College of Radiologists (2017) The breast imaging and diagnostic workforce in the United Kingdom. Available via https://www.rcr.ac.uk/publication/breastimaging-and-diagnostic-workforce-united-kingdom. Accessed 30 Dec 2018

18. Wing P, Langelier MH (2009) Workforce shortages in breast imaging: impact on mammography utilization. Am J Roentgenol 192: 370-378

19. Wallis MG, Moa E, Zanca F, Leifland K, Danielsson M (2012) Two-view and single-view tomosynthesis versus full-field digital mammography: high-resolution X-ray imaging observer study. Radiology 262:788-796

20. Visser R, Veldkamp WJ, Beijerinck D et al (2012) Increase in perceived case suspiciousness due to local contrast optimisation in digital screening mammography. Eur Radiol 22:908-914

21. Hupse R, Samulski M, Lobbes MB et al (2013) Computer-aided detection of masses at mammography: interactive decision support versus prompts. Radiology 266:123-129

22. Gennaro G, Hendrick RE, Ruppel P et al (2013) Performance comparison of single-view digital breast tomosynthesis plus single-view digital mammography with two-view digital mammography. Eur Radiol 23:664-672

23. Siemens Medical Solutions USA Inc (2015) FDA Application: Mammomat Inspiration with Digital Breast Tomosynthesis. Available via https://www.accessdata.fda.gov/cdrh docs/pdf14/ P140011b.pdf. Accessed March 32018 
24. Garayoa J, Chevalier M, Castillo M et al (2018) Diagnostic value of the stand-alone synthetic image in digital breast tomosynthesis examinations. Eur Radiol 28:565-572

25. Rodriguez-Ruiz A, Gubern-Merida A, Imhof-Tas M et al (2017) One-view digital breast tomosynthesis as a stand-alone modality for breast cancer detection: do we need more? Eur Radiol. https:// doi.org/10.1007/s00330-017-5167-3

26. Clauser P, Baltzer PA, Kapetas P et al (2019) Synthetic 2dimensional mammography can replace digital mammography as an adjunct to wide-angle digital breast tomosynthesis. Invest Radiol 54:83-88

27. Blackwelder WC (1982) "Proving the null hypothesis" in clinical trials. Control Clin Trials 3:345-353

28. Chen W, Petrick NA, Sahiner B (2012) Hypothesis testing in noninferiority and equivalence MRMC ROC studies. Acad Radiol 19: $1158-1165$

29. Gallas BD, Bandos A, Samuelson FW, Wagner RF (2009) A framework for random-effects ROC analysis: biases with the bootstrap and other variance estimators. Commun Stat - Theory Methods 38: 2586-2603

30. Gallas B (2017) iMRMC v4.0: application for analyzing and sizing MRMC reader studies. Division of imaging, diagnostics, and software reliability, OSEL/CDRH/FDA, Silver Spring, MD. Available via https://github.com/DIDSR/iMRMC/releases, https:// cran.r-project.org/web/packages/iMRMC/index.html. Accessed 30 Dec 2018

31. Gennaro G (2018) The "perfect" reader study. Eur J Radiol In press

32. Chen W, Gong Q, Gallas BD (2018) Efficiency gain of paired splitplot designs in MRMC ROC studies. Medical imaging 2018: image perception, observer performance, and technology assessment. International Society for Optics and Photonics, pp 105770F

33. Gallas BD, Brown DG (2008) Reader studies for validation of CAD systems. Neural Netw 21:387-397

34. Chen W, Samuelson FW (2014) The average receiver operating characteristic curve in multireader multicase imaging studies. Br J Radiol 87:20140016

35. Dang PA, Freer PE, Humphrey KL, Halpern EF, Rafferty EA (2014) Addition of tomosynthesis to conventional digital mammography: effect on image interpretation time of screening examinations. Radiology 270:49-56

Publisher's note Springer Nature remains neutral with regard to jurisdictional claims in published maps and institutional affiliations. 